\title{
Diferenças entre números fuzzy
}

\author{
Laécio Carvalho de Barros, $\quad$ Francielle Santo Pedro \\ Instituto de Matemática, Estatística e Computação Científica, IMECC, UNICAMP, \\ 13083-970, Campinas, SP \\ E-mail: laeciocb@ime.unicamp.br, fran.stopedro@gmail.com \\ Luciana Takata Gomes \\ Departamento de Física, Química e Matemática, UFSCar, 18052-780, Sorocaba, SP \\ E-mail: lucianatakata@ufscar.br
}

Resumo: Apresentamos diversas definições de diferenças entre números fuzzy presentes na literatura: tradicional, Hukuhara, generalizada de Hukuhara, generalizada, CIA e via distribuições. Exemplificamos através de um problema de transmissão direta e comparamos as diferentes abordagens.

Palavras-chave: Diferenças, números fuzzy.

\section{Introdução}

A diferença tradicional entre números fuzzy, baseada na diferença entre intervalos (ou, equivalentemente, definida via extensão de Zadeh) [1], leva em conta todas as possíveis combinações de elementos entre os dois conjuntos. Como resultado, a resposta é sempre maior em termos de diâmetro do que qualquer um dos conjuntos envolvidos na operação. Assim, a diferença entre dois números não-crisp é sempre um número não crisp e subtrair um número não-crisp dele mesmo nunca é o número zero.

O resultado da subtração de um número não-crisp $A$ dele mesmo utilizando a diferença de Hukuhara $\left(A \Theta_{H} A\right)[7]$, por sua vez, é o número zero. Porém, entre dois números fuzzy distintos $A$ e $B$ ela só está definida quando o primeiro termo possui diâmetro no mínimo igual ao segundo. Generalizando a ideia dessa diferença, $[8,9]$ propuseram a diferença generalizada de Hukuhara, que também possui a propriedade $A-A=0$ mas está definida para uma classe maior de pares de números fuzzy. Outra generalização é a diferença generalizada [2, 9], que possui os mesmos resultados que a generalizada de Hukuhara, quando esta existe, mas que está definida para mais pares de números fuzzy.

Outra proposta é a CIA (constraint interval arithmetic) [6], que admite uma espécie de interação entre os dois números fuzzy envolvidos na subtração. Também levando em contas as interações, é possível definir subtrações entre números fuzzy utilizando a distribuição conjunta de possibilidade (ou pertinência) entre os números fuzzy operados [4].

Neste trabalho apresentamos as definições de todas as diferenças citadas e analisamos suas relações e apresentamos um exemplo ilustrativo.

\section{Conceitos básicos}

Nessa seção apresentamos alguns conceitos básicos e notação utilizados.

Definição 2.1. Um subconjunto fuzzy $A$ de $U$ é caracterizado por uma função de pertinência $\mu_{A}: U \longrightarrow[0,1]$. 
Como um conjunto clássico é definido por sua função característica cujo contradomínio é $\{0,1\}$, podemos dizer então que um conjunto clássico é um caso particular de um conjunto fuzzy. Denotamos por $\mathcal{F}(U)$ a família de todos os subconjuntos fuzzy de $U$.

$U$ será um espaço topológico, a partir de agora.

Definição 2.2. Seja $A$ um subconjunto fuzzy de $U$ e $\alpha \in[0,1]$. O $\alpha$-nivel de $A$ é o subconjunto clássico de $U$ que é definido por

$$
[A]_{\alpha}=\left\{x \in U: \mu_{A}(x) \geq \alpha\right\} \text { para } 0<\alpha \leq 1 .
$$

$O$ nível zero de um conjunto fuzzy $A$ de $U$ é definido como o menor conjunto fechado que contém o suporte do conjunto fuzzy $A$, isto é, $[A]_{0}=\overline{\left\{x \in U: \mu_{A}(x)>0\right\}}$.

A família de todos os subconnjuntos fuzzy de $U$ cujos $\alpha$-níveis são não-vazios, compactos e convexos será denotada por $\mathcal{F}_{\mathcal{C}}(U)$.

Definição 2.3. Um subconjunto fuzzy $A$ é dito ser um número fuzzy quando o conjunto universo no qual $\mu_{A}$ esta definida, é o conjunto dos números reais $\mathbb{R}$ e satisfaz:

i. todos os $\alpha$-níveis de $A$ são intervalos fechados e não vazios de $\mathbb{R}$;

ii. $\left\{x \in U: \mu_{A}(x)>0\right\}$ é um conjunto limitado.

A família dos números fuzzy coincide $\operatorname{com} \mathcal{F}_{\mathcal{C}}(\mathbb{R})$.

Definição 2.4. Uma t-norma $T$ é qualquer operação binária $T:[0,1] \times[0,1] \rightarrow[0,1]$ que possui as seguintes propriedades:

1. elemento neutro: $1 T x=x$;

2. comutativa: $x T y=y T x$;

3. associativa: $x T(y T z)=(x T y) T z$;

4. monotonicidade: se $x \leq u$ e $y \leq v$, então $x T y \leq u T v$.

O princípio de extensão de Zadeh é uma importante ferramenta amplamente empregada para estender funções com valores únicos para funções com valores em conjuntos fuzzy. A definição a seguir é uma definição mais geral, que tem a extensão de Zadeh como caso particular quando a t-norma é a do mínimo.

Definição 2.5. (Princípio de extensão sup-T) Sejam $f$ uma função tal que $f: U \times V \rightarrow Z$ e, $A$ e $B$ subconjuntos fuzzy de $U$ e $V$, respectivamente. Uma extensão de $f$ via $T$ é a função $\widehat{f}$ que, aplicada a $A$ e $B$, fornece o subconjunto fuzzy $\widehat{f}(A, B)$ de $Z$, cuja função de pertinência é dada como segue

$$
\mu_{\widehat{f}(A, B)}(z)=\left\{\begin{array}{cll}
\sup _{f^{-1}(z)} T\left(\mu_{A}(u), \mu_{B}(v)\right) & \text { se } & f^{-1}(z) \neq \emptyset \\
0 & \text { se } & f^{-1}(z)=\emptyset
\end{array}\right.
$$

sendo $f^{-1}(z)=\{(u, v): f(u, v)=z\}$ denomina-se pré-imagem de $z$.

Os conceitos a seguir são provenientes da teoria de possibilidades e serão utilizados para definir diferença interativa entre números fuzzy.

Definição 2.6. Uma distribuição de possibilidade sobre $\Omega \neq \phi$ é uma função $\mu: \Omega \longrightarrow[0,1]$ que satisfaz $\sup _{\omega \in \Omega} \mu(\omega)=1$. 
Definição 2.7. Sejam $A$ e $B$ números fuzzy e $C \in \mathcal{F}_{\mathcal{C}}\left(\mathbb{R}^{2}\right)$, então $\mu_{C}$ é uma distribuição de possibilidade conjunta de $A$ e $B$ se

$$
\max _{y \in \mathbb{R}} \mu_{C}(x, y)=\mu_{A}(x) \quad e \quad \max _{x \in \mathbb{R}} \mu_{C}(x, y)=\mu_{B}(y)
$$

Além disso, $\mu_{A}$ e $\mu_{B}$ são chamadas distribuições marginais de $C$.

A Definição 2.5 pode ser estendida para casos mais gerais considerando-se distribuições de possibilidade conjuntas, das quais as t-normas são casos particulares.

Definição 2.8. Seja $C$ uma distribuição de possibilidade conjunta com distribuições de possibilidades marginais $\mu_{A}$ e $\mu_{B}$, e seja $f: \mathbb{R}^{2} \longrightarrow \mathbb{R}$ uma função contínua. Se $A$ e $B$ são números fuzzy completamente correlacionados, então a extensão de $f$ via $C$ é a função $f_{C}$ cuja função de pertinência é dada por

$$
\mu_{f_{C}(A, B)}(z)=\left\{\begin{array}{ccc}
\sup _{y=f(x, y)} \mu_{C}(x, y) & \text { se } & f^{-1}(z) \neq \emptyset \\
0 & \text { se } & f^{-1}(z)=\emptyset
\end{array}\right.
$$

sendo $f^{-1}(z)=\{(x, y): f(x, y)=z\}$.

Teorema 2.9. [3] Sejam $A, B \in \mathcal{F}(\mathbb{R})$ números fuzzy completamente correlacionados, seja $C$ sua distribuição de possibilidade conjunta e $f: \mathbb{R}^{2} \longrightarrow \mathbb{R}^{2}$ uma função contínua. Então,

$$
\left[f_{C}(A, B)\right]_{\alpha}=f\left([C]_{\alpha}\right) .
$$

Definição 2.10. Dois números fuzzy $A$ e $B$ são declarados completamente correlacionados se existem $q, r \in \mathbb{R}$, com $q \neq 0$, tais que sua distribuição de possibilidade conjunta é definida por

$$
\mu_{C}(x, y)=\mu_{A}(x) \mathcal{X}_{\{q x+r=y\}}(x, y)=\mu_{B}(y) \mathcal{X}_{\{q x+r=y\}}(x, y)
$$

sendo que $\mathcal{X}_{\{q x+r=y\}}(x, y)$ é a função característica da reta $\left\{(x, y) \in \mathbb{R}^{2}: q x+r=y\right\}$.

\section{Diferenças entre números fuzzy}

A seguir apresentamos diversas maneiras presentes na literatura de realizar a diferença entre dois números fuzzy.

Definição 3.1. Suponha $A$ e $B$ dois números fuzzy de $\mathbb{R}$. Seja $f: \mathbb{R}^{2} \rightarrow \mathbb{R}$ definido como $f(x, y)=x-y$, isto é, o operador subtração. A diferença tradicional entre dois números fuzzy é o número fuzzy $f(A, B)=A-B$, cuja função de pertinência é dada por

$$
\mu_{(A-B)}(z)=\left\{\begin{array}{ccc}
\sup _{\phi(z)} \min \left[\mu_{A}(x), \mu_{B}(y)\right] & \text { se } & \phi(z) \neq \emptyset \\
0 & \text { se } & \phi(z)=\emptyset
\end{array},\right.
$$

$\operatorname{sendo} \phi(z)=\{(x, y): x-y=z\}$.

Proposição 3.2. Sejam $A, B$ números fuzzy com $\alpha$-níveis dados, respectivamente, por $\left[a_{\alpha}^{-}, a_{\alpha}^{+}\right]$ $e\left[b_{\alpha}^{-}, b_{\alpha}^{+}\right]$. Então a diferença tradicional entre dois números fuzzy $A$ e $B$ é o número fuzzy $A-B$ cujos $\alpha$-níveis são dados por

$$
[A-B]_{\alpha}=\left[a_{\alpha}^{-}-b_{\alpha}^{+}, a_{\alpha}^{+}-b_{\alpha}^{-}\right]
$$


Definição 3.3. Sejam $A, B$ números fuzzy e $f(x, y)=x-y$ o operador subtração, então a extensão sup $-T$ do número fuzzy $A-B$ é obtida pela seguinte função de pertinência

$$
\mu_{A-B}(z)=\sup _{x-y} T\left(\mu_{A}(x), \mu_{B}(y)\right), z \in \mathbb{R}
$$

Observação 3.4. Se na Definição 3.3 a t-norma utilizada for o mínimo, a diferença resultante é a mesma que a diferença tradicional.

Assim como a Definição 2.8 de extensão via distribuição conjunta generaliza o conceito de extensão via t-norma que por sua vez generaliza a extensão de Zadeh (que é o caso particular em que a distribuição é dada pelo mínimo), a definição de diferença via extensão com a t-norma do mínimo pode ser generalizada pela diferença com t-normas mais gerais que por sua vez são casos particulares de distribuições conjuntas. A seguir definimos esta última diferença.

Definição 3.5. Suponha $A$ e $B$ dois números fuzzy de $\mathbb{R}$. Seja $f: \mathbb{R}^{2} \rightarrow \mathbb{R}$ definida por $f(x, y)=x-y$, isto é, o operador subtração. A diferença via distribuição conjunta $C$ entre dois números fuzzy é o número fuzzy $f(A, B)=A-B$, cuja função de pertinência é definida por

$$
\left.\mu_{(A-J} B\right)(z)=\left\{\begin{array}{lll}
\sup _{(z)} C(x, y) & \text { se } & \phi(z) \neq \emptyset \\
\phi(z) & \text { se } & \phi(z)=\emptyset
\end{array},\right.
$$

sendo $\phi(z)=\{(x, y): x-y=z\}$.

Definição 3.6. [4] A subtração de dois números fuzzy completamente correlacionados $A$ e $B$ é definida por

$$
\mu_{A-{ }_{C} B}(z)=\sup _{z=x-y} \mu_{C}(x, y) .
$$

Isto é, $\mu_{A-C} B(z)=\sup _{z=x-y} \mu_{B}(y) \mathcal{X}_{\{q x+r=y\}}(x, y)$.

Assim, para todo $\alpha \in[0,1]$, temos $\left[A-{ }_{C} B\right]_{\alpha}=(q-1)[B]_{\alpha}+r$.

Observação 3.7. A soma de dois números fuzzy completamente correlacionados $A$ e $B$ é dada por $[A+C B]_{\alpha}=(q+1)[B]_{\alpha}+r$.

Proposição 3.8. Dados dois números fuzzy $A$ e $B$ completamente correlacionados tais que $[A]_{\alpha}=q[B]_{\alpha}+r$, a diferença $A-{ }_{C} B$ possui a seguinte expressão

$$
\left[A-{ }_{C} B\right]_{\alpha}=\left\{\begin{array}{ccc}
i . & {\left[(q-1) b_{\alpha}^{-},(q-1) b_{\alpha}^{+}\right]+r} & \text { se } q>1 \\
\text { ii. } & {\left[(q-1) b_{\alpha}^{+},(q-1) b_{\alpha}^{-}\right]+r} & \text { se } 0 \leq q \leq 1 \\
\text { iii. } & {\left[(q-1) b_{\alpha}^{+}+r,(q-1) b_{\alpha}^{-}+r\right]} & \text { se } q<0 .
\end{array}\right.
$$

Em [5] propôs-se uma aritmética que considera dependência entre números fuzzy, denominada CIA (constraint interval arithmetic). Para tanto ele redefiniu intervalos como funções com valores reais, isto é, um intervalo $\left[a^{-}, a^{+}\right]$é dado pela função $A^{I}\left(a^{-}, a^{+}, \lambda_{A}\right)=\{a: a=(1-$ $\left.\left.\lambda_{A}\right) a^{-}+\lambda_{A} a^{+}, 0 \leq \lambda_{A} \leq 1\right\}$.

Definição 3.9. A subtração entre dois intervalos $A=\left[a^{-}, a^{+}\right]$e $B=\left[b^{-}, b^{+}\right]$é dada por

$$
A-_{C I A} B=\left\{\left[\left(1-\lambda_{A}\right) a^{-}+\lambda_{A} a^{+}\right]-\left[\left(1-\lambda_{B}\right) b^{-}+\lambda_{B} b^{+}\right], 0 \leq \lambda_{A} \leq 1,0 \leq \lambda_{B} \leq 1\right\} .
$$

Lodwick em [6] estendeu essa ideia para o caso fuzzy, baseando-se na afirmação que aritmética de números fuzzy é equivalente a aritmética de intervalos em cada $\alpha$-nível.

Definição 3.10. A subtração entre dois números fuzzy $A$ e $B$ é definida em níveis por

$$
\left[A-_{C I A} B\right]_{\alpha}=\left\{\left[\left(1-\lambda_{A}\right) a_{\alpha}^{-}+\lambda_{A} a_{\alpha}^{+}\right]-\left[\left(1-\lambda_{B}\right) b_{\alpha}^{-}+\lambda_{B} b_{\alpha}^{+}\right], 0 \leq \lambda_{A} \leq 1,0 \leq \lambda_{B} \leq 1\right\} .
$$


Observação 3.11. No caso em que os dois números fuzzy são o mesmo

$$
\begin{aligned}
{\left[A-{ }_{\mathrm{CIA}} A\right]_{\alpha} } & =\left\{\left[\left(1-\lambda_{A}\right) a_{\alpha}^{-}+\lambda_{A} a_{\alpha}^{+}\right] \circ\left[\left(1-\lambda_{A}\right) a_{\alpha}^{-}+\lambda_{A} a_{\alpha}^{+}\right], 0 \leq \lambda_{A} \leq 1\right\} \\
& =\{0\}
\end{aligned}
$$

ou seja, $A-_{\text {CIA }} A=\{0\}$.

Definição 3.12. Dados dois números fuzzy $A, B$ a diferença de Hukuhara (H-deferença) $A \ominus_{H}$ $B=C$ é o número fuzzy $C$ tal que $A=B+C$, se ele existir.

Definição 3.13. [8, 9] Dados dois números fuzzy $A, B$ a diferença generalizada de Hukuhara (diferença gH) $A \Theta_{g H} B=C$ é o número fuzzy $C$, se ele existir, tal que (i) $A=B+C$ ou (i) $B=A-C$.

Definição 3.14. [2, 9] Dados dois números fuzzy $A, B$ a diferença generalizada (diferença g) $A \ominus_{g} B=C$ é o número fuzzy $C$, se ele existir, com $\alpha$-níveis

$$
\left[A \ominus_{g} B\right]_{\alpha}=c l \bigcup_{\beta \geq \alpha}\left([A]_{\beta} \ominus_{g H}[B]_{\beta}\right), \forall \alpha \in[0,1],
$$

onde a diferença $g H\left(\ominus_{g H}\right)$ é acerca dos intervalos $[A]_{\beta}$ e $[B]_{\beta}$.

\section{Resultados e Aplicações}

A seguir faremos as comparações entre as diferenças fuzzy mas antes definiremos a seguinte notação: $X_{\text {diferença }}$ é o conjunto de pares de números fuzzy tal que a diferença entre eles existam.

Diâmetro das diferenças:

- $\operatorname{diam}\left(\left[A \ominus_{H} B\right]_{\alpha}\right) \leq \operatorname{diam}\left(\left[A-_{T} B\right]_{\alpha}\right) \leq \operatorname{diam}\left([A-B]_{\alpha}\right)$;

- $\operatorname{diam}\left(\left[A \ominus_{g H} B\right]_{\alpha}\right) \leq \operatorname{diam}\left(\left[A-{ }_{T} B\right]_{\alpha}\right) \leq \operatorname{diam}\left([A-B]_{\alpha}\right)$;

- $\operatorname{diam}\left(\left[A \ominus_{g} B\right]_{\alpha}\right) \leq \operatorname{diam}\left(\left[A-{ }_{T} B\right]_{\alpha}\right) \leq \operatorname{diam}\left([A-B]_{\alpha}\right)$;

- $\operatorname{diam}\left(\left[A-{ }_{J} B\right]_{\alpha}\right) \leq \operatorname{diam}\left([A-B]_{\alpha}\right)$;

- $\operatorname{diam}\left([A-C B]_{\alpha}\right) \leq \operatorname{diam}\left([A-B]_{\alpha}\right)$;

- $\operatorname{diam}\left(\left[A-_{\mathrm{CIA}} B\right]_{\alpha}\right) \leq \operatorname{diam}\left([A-B]_{\alpha}\right)$.

Existência das diferenças:

- $X_{\text {tradicional }}, X_{\mathrm{CIA}}, X_{T}, X_{J}$ e $X_{C}$ equivalem a $\mathcal{F}_{\mathcal{C}}(\mathbb{R}) \times \mathcal{F}_{\mathcal{C}}(\mathbb{R})$, ou seja, nesses casos a diferença entre dois números fuzzy sempre existe;

- $X_{T} \subseteq X_{\text {tradicional }}$;

- $X_{\text {CIA }} \subset X_{\text {tradicional }}$;

- $X_{C} \subseteq X_{J} \subset X_{T}$

- $X_{H} \subset X_{g H} \subset X_{g} \subset X_{T}$ e as diferenças H, gH e g nem sempre existem;

Observação 4.1. Note que a existência da diferença via distribuições conjuntas é determinada pela existência da mesma. 


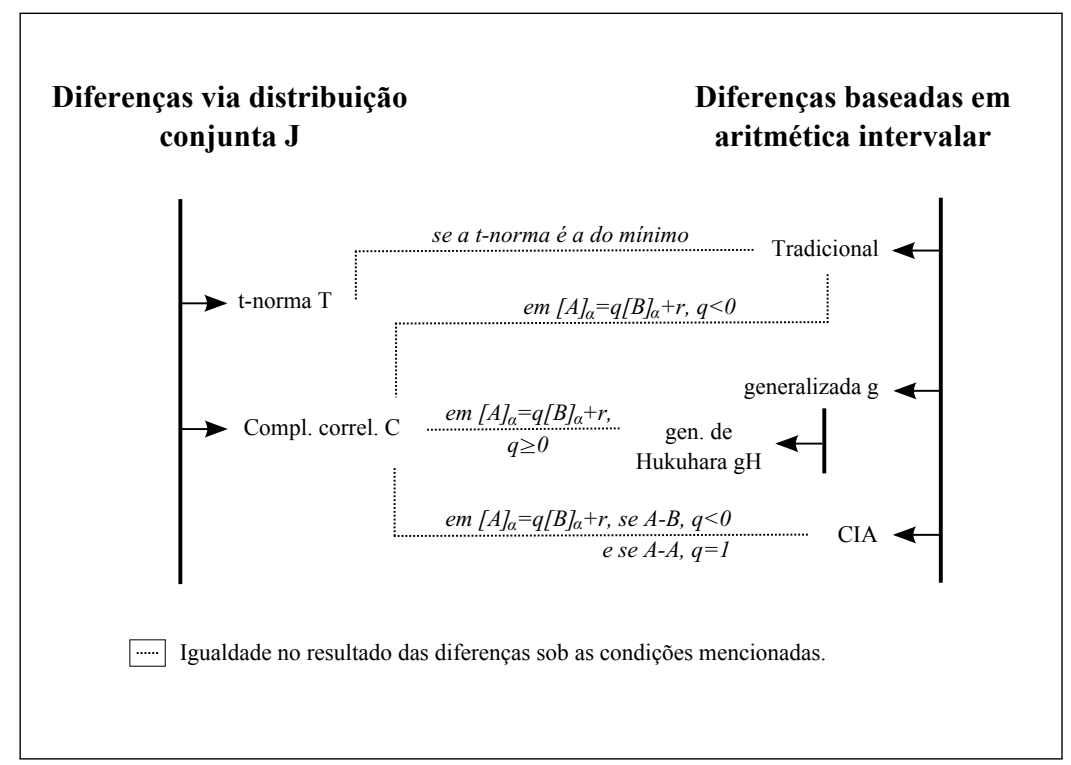

Figura 1: Relações entre diferenças definidas via distribução conjunta e diferenças baseadas em aritmética intervalar. As linhas pontilhadas significam igualdade nos resultados das subtrações entre dois números fuzzy, considerando-se as hipóteses mencionadas.

Como na diferença completamente correlacionada temos

$$
\left[A-{ }_{C} B\right]_{\alpha}=\left\{\begin{array}{clc}
i . & {\left[a_{\alpha}^{-}-b_{\alpha}^{-}, a_{\alpha}^{+}-b_{\alpha}^{+}\right]} & \text {se } q \geq 1 \\
i i . & {\left[a_{\alpha}^{+}-b_{\alpha}^{+}, a_{\alpha}^{-}-b_{\alpha}^{-}\right] \quad \text { se } 0 \leq q \leq 1} \\
\text { iii. } & {\left[a_{\alpha}^{-}-b_{\alpha}^{+}, a_{\alpha}^{+}-b_{\alpha}^{-}\right] \quad \text { se } q<0}
\end{array}\right.
$$

sabemos que se $q \geq 1$ a diferença $C$ é a diferença de Hukuhara, se $q \geq 0$ a diferença $C$ é a diferença generalizada de Hukuhara e para $q<0$ a diferença $C$ é a diferença tradicional. Comparando essa diferença com a CIA, notamos que elas são equivalentes se e somente se $q=1$ quando calculamos $A-{ }_{C} A$ e $q<0$ se computamos $A-{ }_{C} B$.

Considere um modelo epidemiológico de transmissão direta com dois compartimentos suscetível-infectado, por exemplo $S I$ ou $S I S$ sem dinâmica vital. Dessa forma, a proporção de suscetíveis $(S)$ somada a proporção de infectados $(I)$ é igual a 1, isto é equivalente a, $I_{t}=1-S_{t}, \forall t$. Suponha que $S$ seja um número fuzzy triangular $(a ; b ; c)$, onde fixado $\bar{t}$ te$\operatorname{mos},\left[S_{\bar{t}}\right]_{\alpha}=\left[s_{\alpha}^{-}, s_{\alpha}^{+}\right]=[(b-a) \alpha+a,(b-c) \alpha+c], 0<a<b<c<1$.

Calcularemos $I_{\bar{t}}$, utilizando cada umas das diferenças entre 1 e $S_{\bar{t}}$ dadas na seção anterior.

- Se $S_{\bar{t}}$ e $I_{\bar{t}}$ são não interativos a diferença entre eles é dada de forma tradicional: $\left[I_{\bar{t}}\right]_{\alpha}=$ $\left[1-s_{\alpha}^{+}, 1-s_{\alpha}^{-}\right]$.

- Se a diferença é dada por $T$-normas (diferentes da $T$-norma do mínimo), ou seja $S_{\bar{t}}$ e $I_{\bar{t}}$ interativos, teremos $\left[I_{\bar{t}}\right]_{\alpha}=\bigcup_{T(\xi, \eta) \geq \alpha}[1]_{\xi}-\left[S_{\bar{t}}\right]_{\eta}, \alpha \in(0,1]$.

- Se $S_{\bar{t}}$ e $I_{\bar{t}}$ são completamente correlacionados, temos que $\left[I_{\bar{t}}\right]_{\alpha}=1-\left[S_{\bar{t}}\right]_{\alpha}$, com $q=-1$ e $r=1$, daí $\left[I_{\bar{t}}\right]_{\alpha}=\left[1-s_{\alpha}^{+}, 1-s_{\alpha}^{-}\right]$. Neste caso $\left[I_{\bar{t}}\right]_{\alpha}+{ }_{C}\left[S_{\bar{t}}\right]_{\alpha}=1$.

- A diferença de Hukuraha não existe, pois não temos diâmetro crescente. Por outro lado, as diferenças $\ominus_{g}$ e $\ominus_{g H}$, são tais que $\left[I_{\bar{t}}\right]_{\alpha}=\left[1-s_{\alpha}^{+}, 1-s_{\alpha}^{-}\right]_{\alpha}$.

- Agora se utilizarmos a $\left({ }_{\text {CIA }}\right)$, obteremos $\left[I_{\bar{t}}\right]_{\alpha}=\left\{1-\left[\left(1-\lambda_{S}\right) s_{\alpha}^{-}+\lambda s_{\alpha}^{+}\right], 0 \leq \lambda_{S} \leq 1\right\}=$ $\left\{1-\left[\left(1-\lambda_{S}\right)((b-a) \alpha+a)+\lambda((b-c) \alpha+c)\right], 0 \leq \lambda_{S} \leq 1\right\}$ que é equivalente ao número triangular $(1-c ; 1-b ; 1-a)$.

Em suma, do ponto de vista de coerência, a diferença que melhor representa o modelo $S I$ é a diferença completamente correlacionada, pois só nesse caso, $S+I=1$, que vem de encontro com a hipótese de não haver dinâmica vital. 


\section{Agradecimentos}

Os dois primeiros autores gostariam de agradecer ao $\mathrm{CNPq}$ pelo auxílio financeiro (processo $305862 / 2013-8$ e $141085 / 2014-2$, respectivamente).

\section{Referências}

[1] L. C. Barros, R. C. Bassanezi, "Tópicos em lógica fuzzy e biomatemática", IMECCUnicamp, Campinas, 2010.

[2] B. Barnabás, L. Stefanini, "Generalized differentiability of fuzzy-valued functions", Fuzzy Sets and Systems, 230 (2013) 119-141.

[3] V. M. Cabral, "Equações diferenciais fuzzy com parâmetros interativos", Tese de Doutorado, IMECC-Unicamp, 2011.

[4] C. Carlsson, R. Fúller, P. Majlender, Additions of completely correlated fuzzy numbers, em "IEEE International Conference on Proceedings" (Fuzzy Systems), pp.535-539, Budapest, Julho (2004).

[5] W. A. Lodwick, "Constrained interval arithmetic", University of Colorado at Denver, Center for Computational Mathematics, 1999.

[6] W. A. Lodwick, E. A. Untiedt, A comparison of interval analysis using constraint interval arithmetic and fuzzy interval: analysis using gradual numbers, em "Annual Meeting of the North American-NAFIPS" (IEEE), 2008.

[7] M. L. Puri, D. A. Ralescu. "Differentials of fuzzy functions", Journal of Mathematical Analysis and Applications, 91.2 (1983) 552-558.

[8] L. Stefanini, B. Barnabás, "Generalized Hukuhara differentiability of interval-valued functions and interval differential equations", Nonlinear Analysis: Theory, Methods $\&$ Applications, 71.3 (2009) 1311-1328.

[9] L. Stefanini, "A generalization of Hukuhara difference and division for interval and fuzzy arithmetic", Fuzzy sets and systems, 161.11 (2010): 1564-1584.

8 (1965) 338-353. 